\title{
Design modification on Indian Road Vehicles to Reduce Aerodynamic Drag
}

\author{
P.Vinayagam*, M.Rajadurai, K.Balakrishnan, G.Mohana Priya \\ Department of Aeronautical Engineering, Mahendra Engineering College, Namakkal, Tamil Nadu, India
}

\begin{abstract}
Reducing vehicle fuel consumption has become one of the most important issues in recent years. Aerodynamic drag contributes to 50-60\% of fuel consumption in trucks on highways. Vehicle aerodynamic performance is mainly determined by drag coefficient, which directly affects engine requirements and fuel consumption. It's well known that drag changes in a crosswind compared with a condition without a crosswind, and that the change depends on the vehicle shape. Pressure drag, a major drag for trucks as they run at lower speeds is produced by the shape of the object. Therefore, addition of some components can suffice the need. The vehicle has been designed by using Catia and then analysed with CFD. The values are compared and the resultant drag reduction is calculated.
\end{abstract}

Keywords-Aerodynamics drag, CATIA, CFD, fuel consumption.

\section{INTRODUCTION}

Vehicles with an aerodynamic shape use less fuel. Air flows easily over them and less energy is needed to move them forward. At $95 \mathrm{Km} / \mathrm{h} 60-70 \%$ of a vehicle's energy is used to move it through the air, compared with only $40 \%$ at $50 \mathrm{Km} / \mathrm{h}$.installing a sloping front roof on a lorry could save you as much as $7 \%$ of your fuel costs. Even small changes to design and shape will make a difference. Take a look at the Aerodynamic checklist, walk around your vehicle and look at each feature to see what improvements you can make. This document covers the aerodynamic styling of commercial vehicles. Vehicles that travel at higher speeds and for longer distances will benefit most from aerodynamic styling, giving you greater savings. Drag is the energy lost pushing through air, and it accounts for most of the fuel used on longdistance journeys, regardless of vehicle type. Overcoming drag uses approx. $60 \%$ of fuel used at cruising speeds when loaded, $70 \%$ when empty. Sharp corners, racks and parts that stick out will add "parasitic drag", further reducing fuel efficiency. Fuel consumption due to aerodynamic drag consumed more than half of the vehicle's energy. Thus, the drag reduction program is one of the most interesting approaches to cater this matter. Aerodynamic drag consists of two main components: skin friction drag and pressure drag. Pressure drag accounts for more than $80 \%$ of the total drag and it is highly dependent on vehicle geometry due to boundary layer separation from rear window surface and formation of wake region behind the vehicle. The location of separation determines the size of wake region and consequently, it determines the value of aerodynamic drag. According to the aerodynamic drag of a road vehicle is responsible for a large part of the vehicle's fuel consumption and contributes up to $50 \%$ of the total vehicle fuel consumption at highway speeds. Reducing the aerodynamic drag offers an inexpensive solution to improve fuel efficiency and thus shape optimization for low drag becomes an essential part of the overall vehicle design process. It has been found that $40 \%$ of the drag force is concentrated at the rear of the geometry.

Investing in good aerodynamic styling on new trucks will repay your investment. Manufacturers go to enormous expense using wind tunnels to improve aerodynamic stability and reduce parasitic drag. The truck pictured is an example of good aerodynamic styling and air management. To understand how this styling reduces your fuel consumption look at the simplified diagram below of an articulated truck without any curved edges. Also note that there is a large gap between the tractor and the trailer. Compare this with the well styled tractor and trailer in the second picture. You'll see that there are fewer areas of turbulence and turbulence causes drag. The second vehicle will use less fuel. Ensure that air can flow easily and smoothly over the shape of your vehicle, by minimizing things that stick out and block the air's passage. Wherever possible chose smooth sided designs, curved edges, hidden buckles and a close gap between your tractor and your trailer.
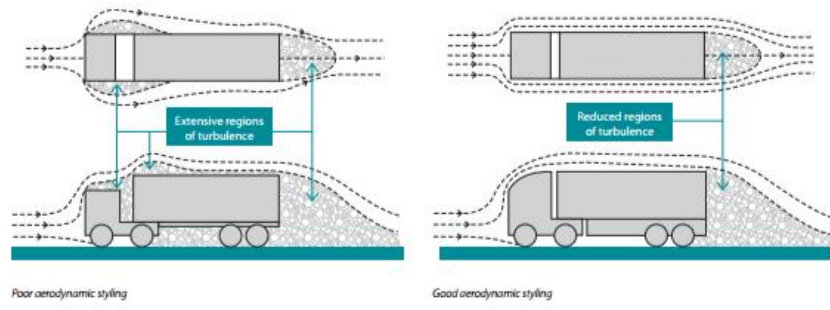

Fig.1: Flow over on a vehicle 


\section{INVERSTIGATION METHOD}

There are various approaches of passive devices to reduce drag. These are front screens, rear screens, structural elements that localize the area of flow detachment (edging), vortex air flow generators, deflectors located over the rear part of vehicle's roof, four-element rear fairing and its components, front fairing.

\section{AERODYNAMICAL DIFFERENCE}

Truck:

$>$ Bluff bodies

$>$ Large viscous regions

$>$ Low aspect ratio (3D)

$>$ Strong interaction between body parts

$>$ Ground effects

Airplane:

$>$ Streamlined

$>$ Inviscid flow dominates

$>$ High aspect ratio (partly 2D)

$>$ Step-by step optimization

\section{CHARECTERISTICS OF FLOW PASSED VEHICLE BODIES}

FRONT: stagnation point, overpressure, accelerating flow SIDE WALLS \& ROOFS: boundary layer separation depending on the rounding up of leading edges around the front.

REAR WALL: In separation bubble nearly constant pressure below the ambient, strong turbulent mixing UNDERBODY GAP: surrounded by rough and moving surfaces, decreasing velocities downstream, sideward outflow.

\section{CAUSE OF DRAG FORCES AT STREAMLINED AND BLUFF BODIES}

Streamlined bodies are characterized by attached flow. The share of pressure forces in drag force (component of aerodynamic force parallel to undisturbed flow) is small. Drag is caused mainly by shear stresses. Since shear forces are small coefficient of drag is relatively small.

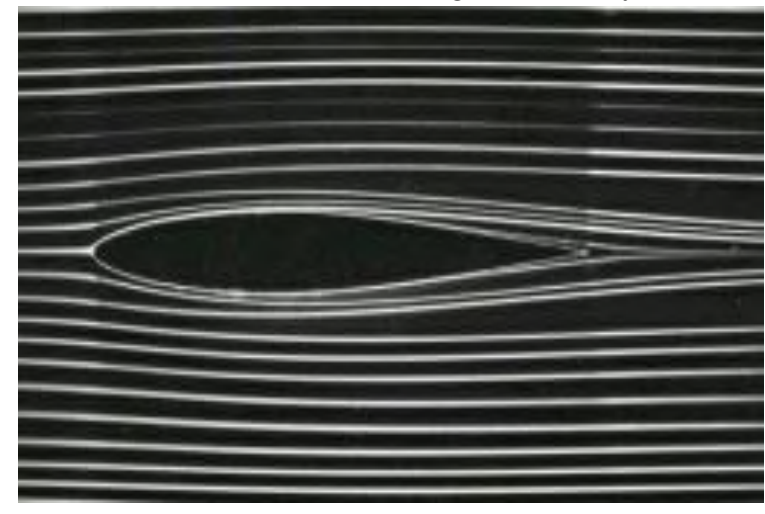

Fig.2: Flow over stream body
Bluff bodies are characterized by boundary layer separation and separation bubbles. Drag is caused mainly by pressure forces, since $\mathrm{p}-\mathrm{p} 0>>\tau$ coefficient of drag is relatively big.

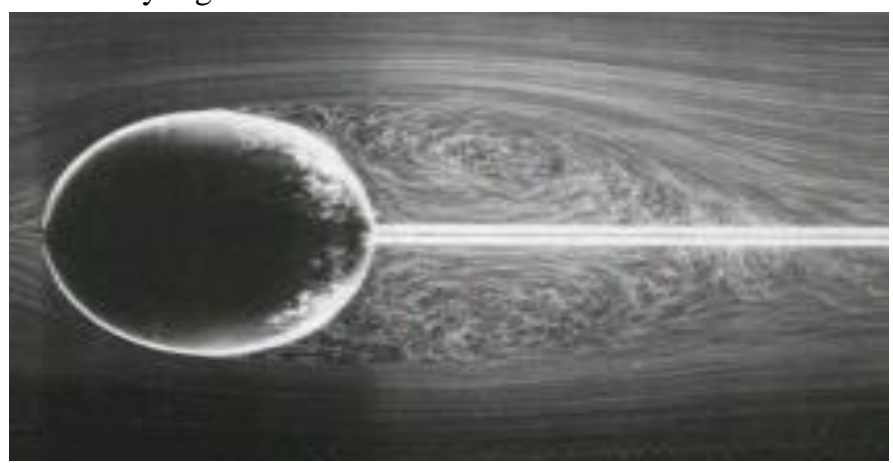

Fig.3: Flow over bluff body

\section{DESIGNING OF TRUCKS}

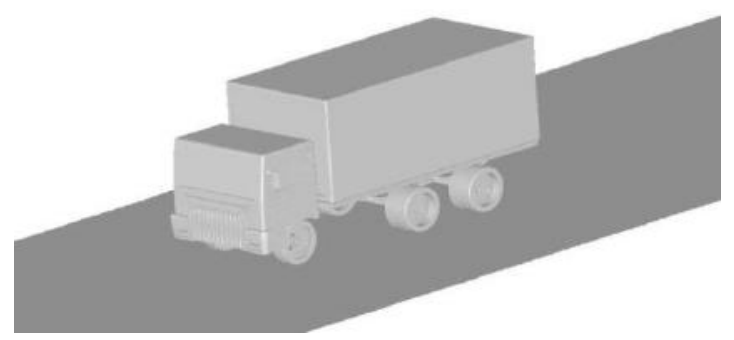

Fig.4: Catia diagram of normal truck

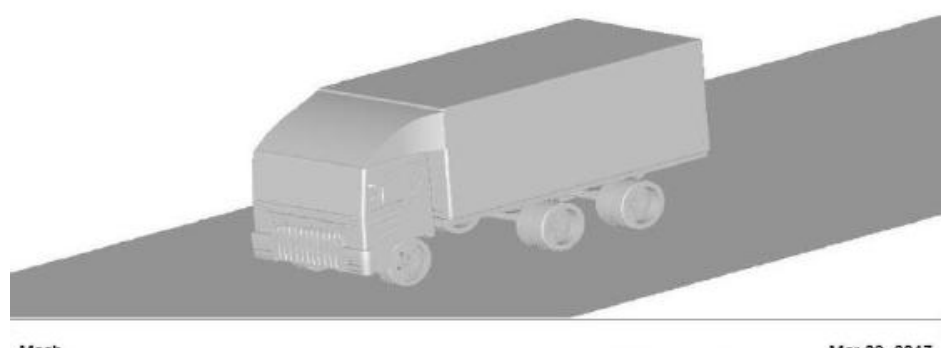

Fig.5: Catia diagram of Aerodynamic truck

\section{MESHING}

FLUENT requires high quality mesh to avoid numerical diffusion. Several mesh quality metrics are involved in order to quantify the quality; however the skewness is the primary metric however the skewness is the primary metric. The aspect ratio and cell size change mesh metrics are also very important. In worst scenarios and depending on the solver used (density based or pressure based) FLUENT can tolerate poor mesh quality. However some applications may require higher mesh quality, resolution and good mesh distribution. The location of poor quality elements helps determine their effect. Some overall mesh quality metrics may be obtained in annoys meshing under the statistics object. Additional mesh quality metrics may be retrieved in FLUENT GUI under mesh/info/quality 
from the menu, or using the TUI commands 'mesh/quality'.

\section{MESH QUALITY REQUIREMENTS FOR FLUENT}

The most important mesh metrics for fluent are, skewness, aspect ratio cell size change (not implemented in ansys meshing). For most applications, poor mesh quality may lead to inaccurate for most applications, for skewness: for hexa, tri and quad it should be less than 0.8 for tetrahedral: it should be less than 0.9 lead to inaccurate solution and/or slow convergence for tetrahedral. It should be less than 0.9. For aspect ratio: it should be less than 40 , but this depends on the flow characteristics. Some applications may require even lower skewness than the suggested valve the flow characteristics more than 50 may be tolerated at the inflation layers. For cell size change suggested valve A- 8 it should be between 1 and 2 .

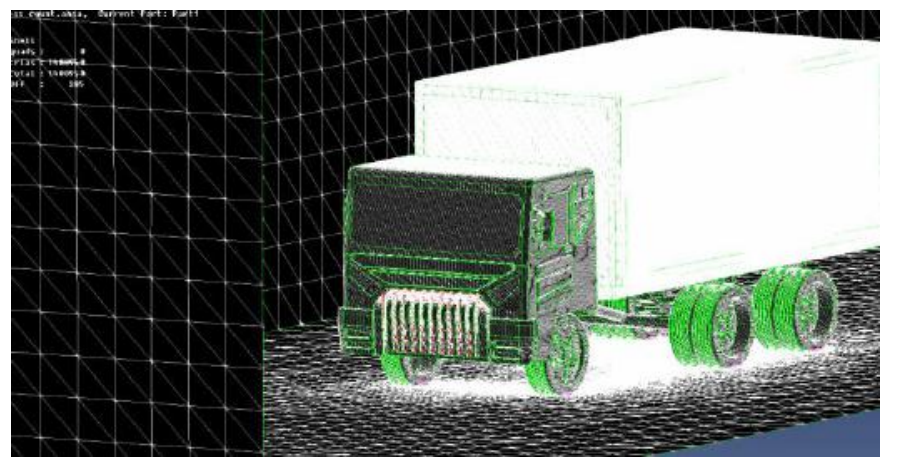

Fig.6: Meshing of Truck

Meshing quadrilateral mesh

No. of nodes: 26738

No. of elements: 110517

\section{RESULTS AND DISCUSSIONS}

The analyzed result of truck in ansys is shown below. From this analyzed result we will be able to understand that where will be the maximum amount of force and parameters are acting.

\section{a. VELOCITY DISTRIBUTIONS ON TRUCKS}

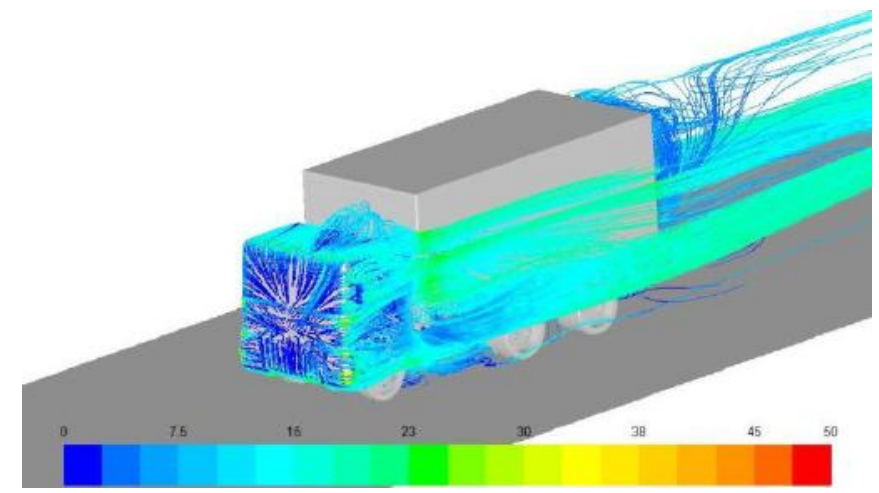

Fig.7: velocity path lines truck 1

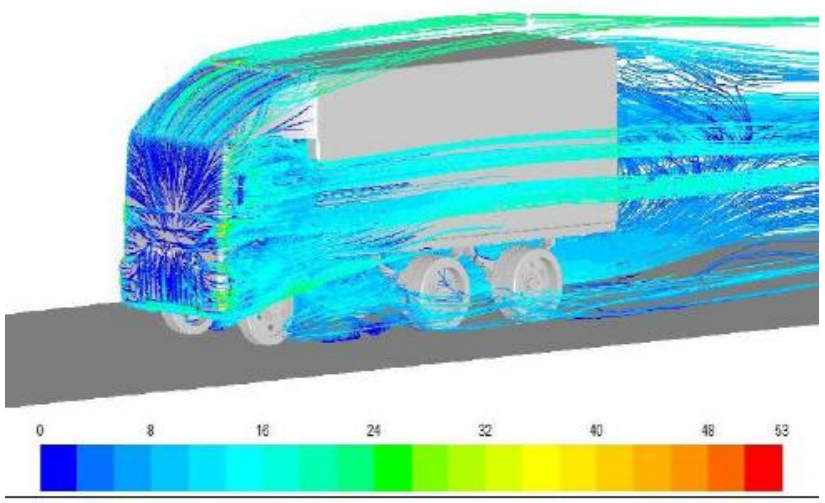

Pathlines Colored by Velocity Magnitude (m/s)

Mar 28, 2017

Fig.8: velocity path lines truck 2

The figure above makes use of streamlines to indicate how the air flows over the trucks. In comparison of two trucks the air flow of modified truck is smoother than the normal truck

\section{b. PRESSURE DISTRIBUTIONS ON TRUCKS}

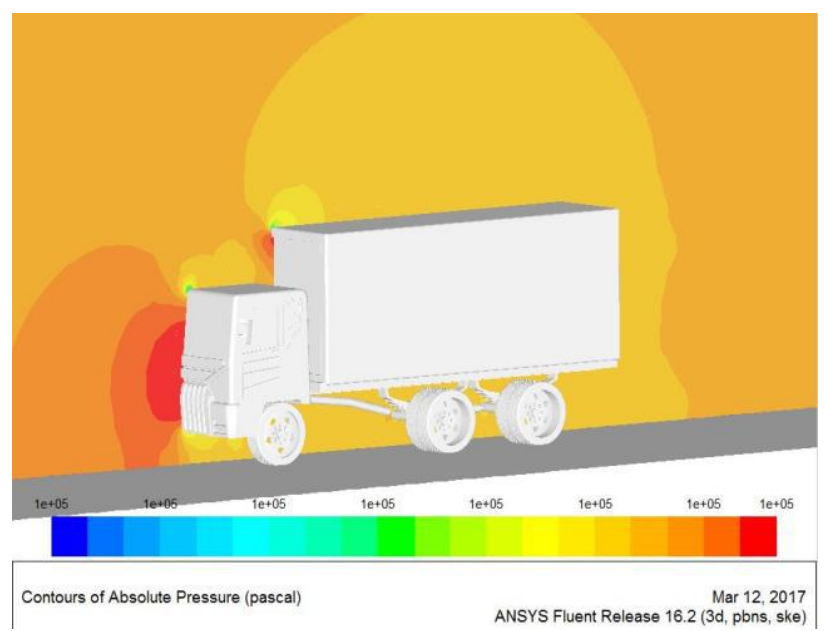

Fig.9: Absolute pressure contour truck 1

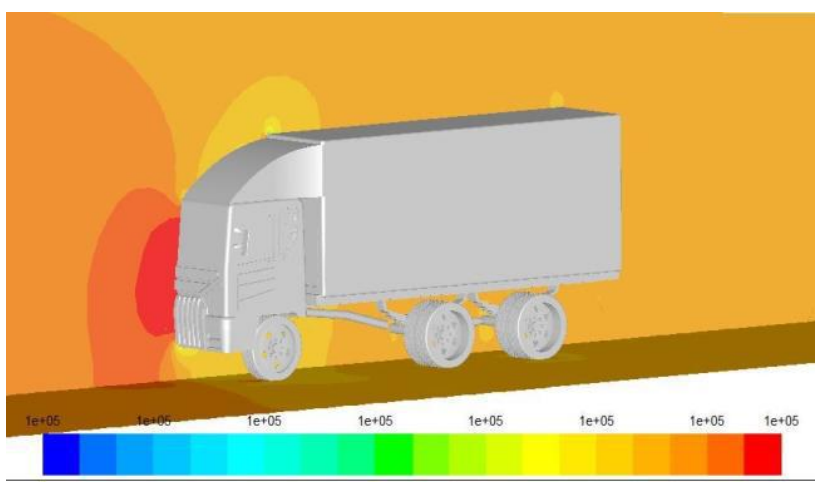

Contours of Absolute Pressure (pascal)

Mar 28, 2017

Fig.10: Absolute pressure contour truck 2 


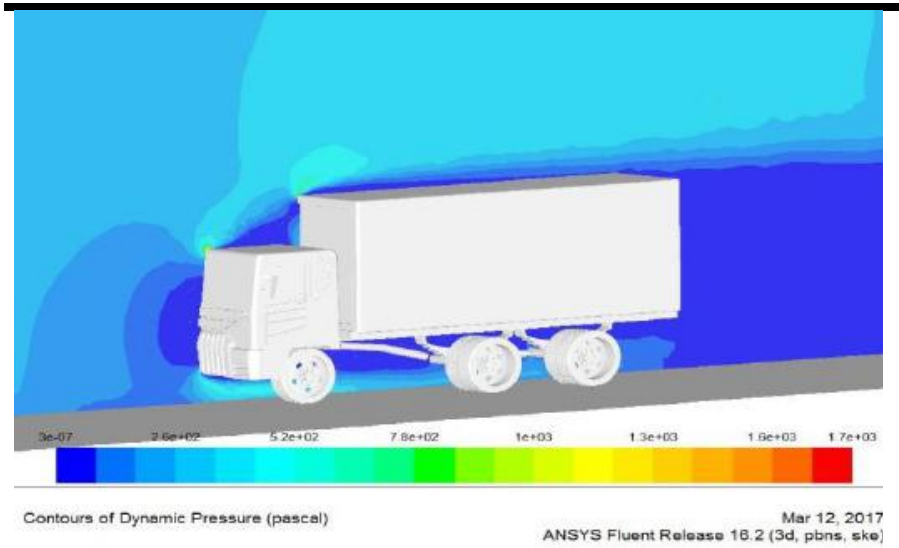

Fig.11: Dynamic pressure contour truck 1

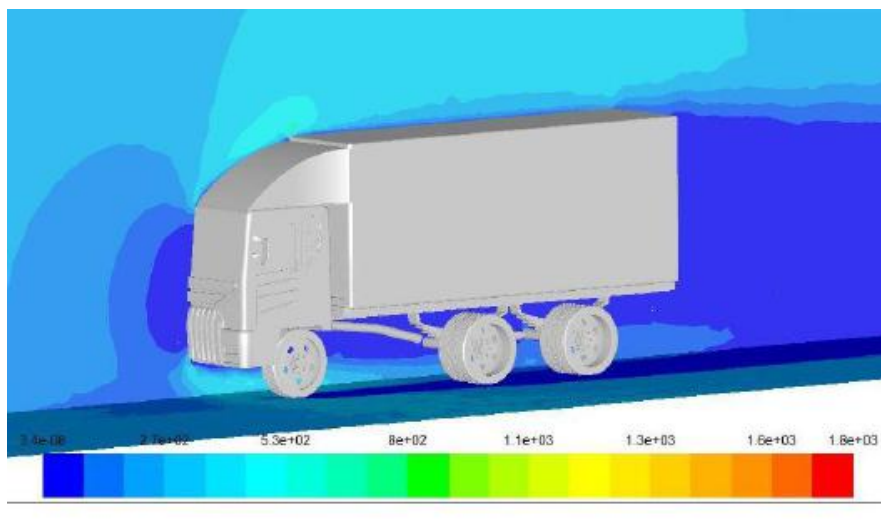

Contours of Dynamic Pressure (pascal)

Mar 28, 2011

Fig.12: Dynamic pressure contour truck 2

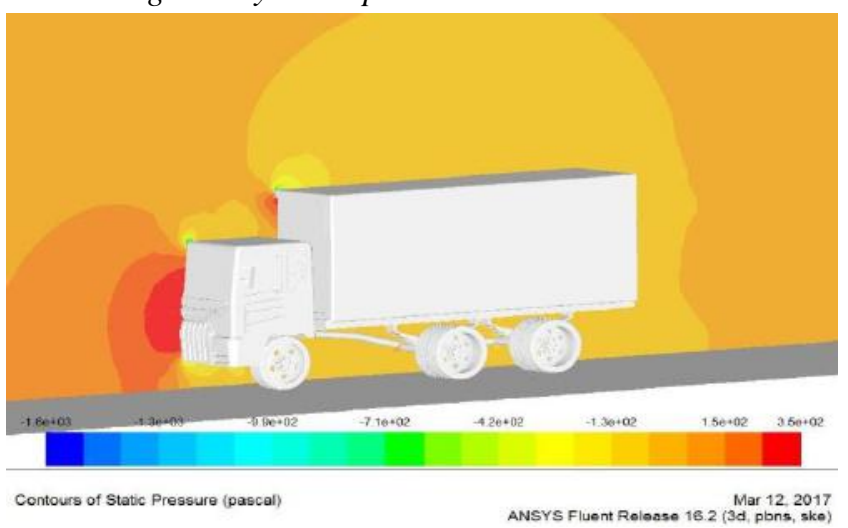

Fig.13: Static pressure contour truck 1

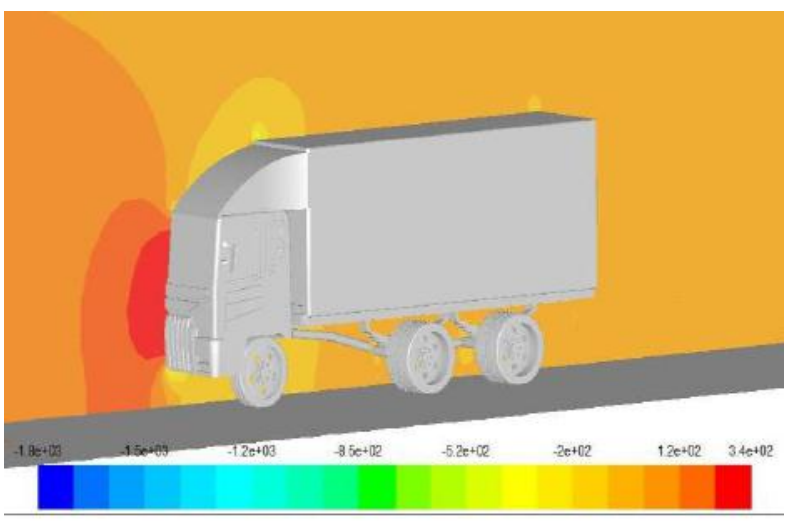

Contours of Static Pressure (pascal)

Mar 28, 2017

Fig.14: Static pressure contour truck 2 c. VELOCITY MAGNITUDE AROUND THE TRUCKS

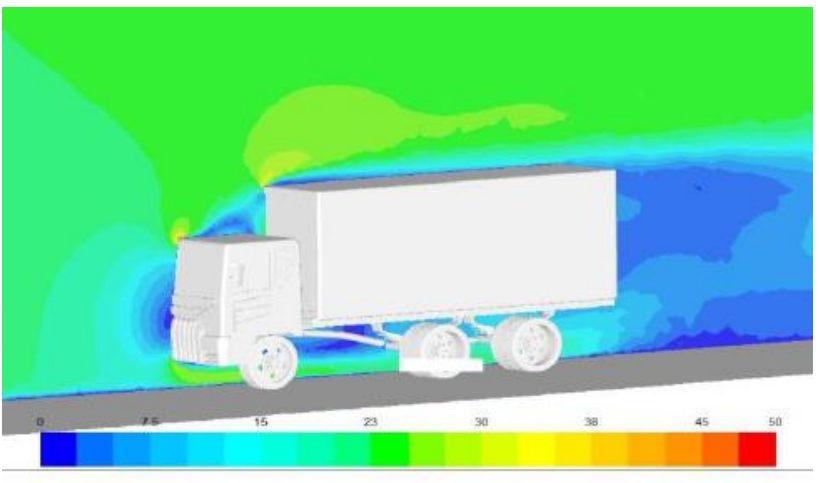

Contours of Velocity Magnitude (m/s)

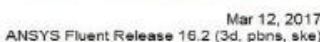

Fig.15: velocity magnitude truck 1

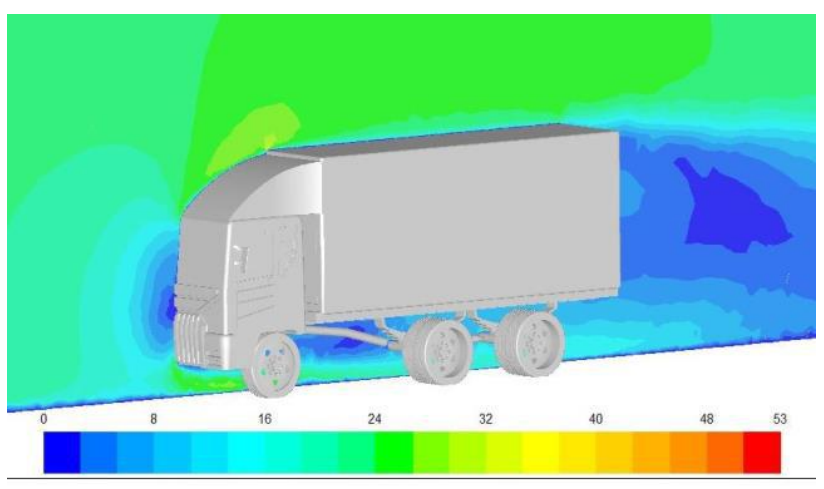

Contours of Velocity Magnitude $(\mathrm{m} / \mathrm{s})$

Mar 28, 2017

Fig.16: velocity magnitude truck 2

\section{COMPARATIVE DATA OF BOTH} MODELS

The result we got from the analysis of the modified model and the existing model are plotted below here we made a comparison between the two design. The plotted diagrams and charts will prove it properly. From the analyzing of the two models we came to know that our modified truck model is give low aerodynamic drag and fuel consumption when we are comparing to the normal truck.

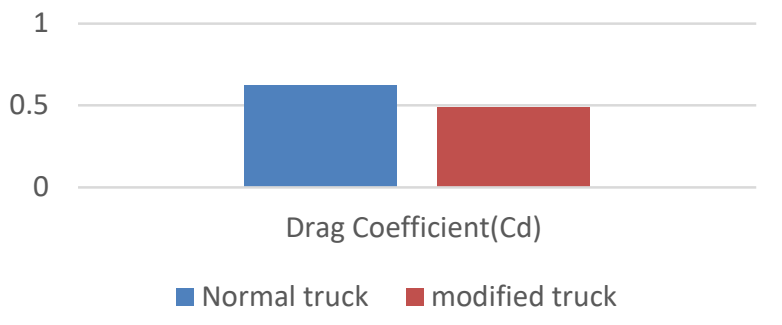

\section{SUGGESTION FOR FUTURE WORKS}

In this project we came across ideas to decrease the aerodynamics drag and to increase the performance of the truck. Here we took the model of normal truck and we succeed in it by changing the Indian normal truck into an aerodynamic one. As a result of that the drag acting on 
the modified truck becomes less, and also the fuel consumption reduced.

So in our project we are only aim to improve the speed and to decrease the drag acting on the truck. But in the same truck there is great chance to reduce the fuel consumption of the truck. The fuel consumption of the truck is mainly depends upon its shape. If somebody is interested to do more future works in this Indian road vehicle we will suggest you to go on with different shapes of Indian road vehicles. Because it is very useful to peoples who using an Indian road vehicles like vans, cars, and bus.

\section{CONCLUSION}

In this project we have designed and analyzed the normal truck and modified truck for reducing aerodynamics drag and fuel consumptions. The comparison of two trucks, the normal truck had high aerodynamics drag than modified truck. The CFD analysis for these trucks studied and gives the results of velocity, pressure, coefficient of drag. From these results the aerodynamics drag produced by our modified truck is low than existing design. The aerodynamic shape of a vehicle is crucial because it has a large impact on fuel. When buying a new vehicle, carefully consider the impact of aerodynamic features. Remember that time invested in this area will be worth the investment.

\section{REFERENCES}

[1] R.H. Barnard (2001):"Road Vehicle Aerodynamic Design, 2nd edition". MechAero Publishing, 2001.

[2] H. Götz and G. Mayr, Aerodynamics of Road Vehicles (Ed. W.H. Hucho), SAE International, pp. 415-488, 1998.

[3] Braus, M., Lanfrit, M. (2001). Simulation of the Ahmed Body. 9th ERCOFACT/IAHR Workshop on Refined Turbulence Modelling.

[4] Guo, L., Zhang, Y., \& Shen, W. (2011). Simulation Analysis of Aerodynamics Characteristics of Different Two-Dimensional Automobile Shapes.

[5] Gustavsson, T. (2006). Alternative approaches to rear end drag reduction. KTH, Department of Aeronautical and Vehicle Engineering, Royal Institute of Technology, TRITA-AVE 2006:12.

[6] H. Lienhart and S. Becker. (2003). Flow and turbulent structure in the wake of a simplified car model. SAE Paper 2003-01-0656, 2003.

[7] Han, T., Hammond, D. C., and SAGI, C. J. (1992). Optimization of bluff body for minimum drag in ground proximity. AIAA Journal, Vol. 30, No. 4, pp. 882-889.

[8] Hu, X., Zhang, R., Ye, J., Yan, X., and Zhao, Z. (2011). Influence of Different Diffuser Angle on
Sedan's Aerodynamic Characteristics. Physics Procedia, Volume 22, Pages 239-245 